\title{
On the Fermi Approximation in Thermal Neutron Scattering*
}

\author{
G. C. Summerfield \\ Department of Nuclear Engineering, The University of Michigan, Ann Arbor, Michigan
}

The Fermi approximation and all higher order corrections are derived for the cross section for thermal neutron scattering from aggregates of atoms. The first order correction is shown to reduce to the result obtained by Lippmann and Schwinger in the special case that the scatterer is a single bound proton. The magnitude of the correction is estimated for an arbitrary number of scattering atoms, and it is shown to be approximately proportional to the inverse of the separation between atoms.

\section{INTRODUCTION}

The Fermi approximation (1) has been used exclusively in calculations of the interactions of thermal neutrons with atoms in matter. However, the conditions for the validity of this approximation are somewhat vague. It has not been tested theoretically except in very special cases, and it has not been deduced in a systematic way for an arbitrary number of atoms in the scatterer. In particular, it has not been clear how the Fermi approximation should be altered to include multiple scattering effects.

In the following work, the Fermi approximation is shown to be the first term in a series expansion for the $T$-matrix, and the higher order corrections are explicitly given. The first order correction is shown to reduce to the result obtained by Lippmann and Schwinger (2) for the special case that the scatterer is a single bound proton. The magnitude of the first order correction is estimated for an arbitrary number of atoms in the scatterer. As we would expect from intuitive arguments, the correction, as estimated, is proportional to the inverse of the separation between atoms.

\section{THE SERIES APPROXIMATION}

Consider a system composed of $N$ atoms at positions $\mathbf{r}_{1}, \cdots, \mathbf{r}_{N}$, and a neutron at position $\mathbf{r}_{n}$. The Hamiltonian for this system is assumed to be the following:

$$
\mathfrak{H C}=\frac{\mathbf{p}_{n}{ }^{2}}{2 m}+\mathfrak{H}_{A}+\sum_{\alpha=1}^{N} V_{\alpha}\left(\mathbf{r}_{n}-\mathbf{r}_{\alpha}\right)
$$

* Work supported by the U. S. Atomic Energy Commission. 
where $\mathbf{p}_{n}$ is the neutron momentum; $\mathcal{H}_{A}$ is the Hamiltonian for the atoms, including interactions between atoms; $m$ is the neutron mass; and $r_{\alpha}\left(\mathbf{r}_{n}-\mathbf{r}_{\alpha}\right)$ is the interaction between the neutron and the nucleus of the atom at $r_{\alpha}$. We label the eigenfunctions of $\mathbf{p}_{n}{ }^{2} 2 m$ and $\mathfrak{H}_{1}$ as:

$$
\begin{aligned}
\left(\mathbf{p}_{n}{ }^{2} / 2 m\right) \psi_{j}\left(\mathbf{r}_{n}\right) & =E_{j} \psi_{j}\left(\mathbf{r}_{n}\right) \\
\mathcal{H}_{.1} \varphi_{l}\left(\mathbf{r}_{1}, \cdots, \mathbf{r}_{N}\right) & =E_{l \varphi_{l}}\left(\mathbf{r}_{1}, \cdots, \mathbf{r}_{N}\right)
\end{aligned}
$$

also, we define:

$$
\mathfrak{H}_{0}=\mathbf{p}_{n}^{2} / 2 m+\mathfrak{H}_{1}
$$

The differential cross section for a neutron scattered from the atoms is:

$$
\frac{d \sigma_{n i}}{d \Omega}=\left(\frac{m}{2 \pi \hbar^{2}}\right)^{2} \frac{l_{j}}{h_{i}}\left|T_{j i}\right|^{2}
$$

where $\hbar \mathbf{k}_{i}$ and $\hbar \mathbf{k}_{f}$ are the initial and final neutron momenta respectively: and $T_{j i}$ is:

$$
T_{j i}=\left\langle\psi_{f}\left(\mathbf{r}_{n}\right) \varphi_{f}\left(\mathbf{r}_{1}, \cdots, \mathbf{r}_{N}\right)|T| \psi_{i}\left(\mathbf{r}_{n}\right) \varphi_{i}\left(\mathbf{r}_{1}, \cdots, \mathbf{r}_{N}\right)\right\rangle
$$

and $T$ is the solution of the following integral equation.

$$
T=r+r i r T
$$

where

$$
\begin{aligned}
& Y^{r}=\sum_{\alpha=1}^{N} V_{\alpha}\left(\mathbf{r}_{n}-\mathbf{r}_{\alpha}\right) ; \\
& G^{\prime}=\left(E^{\prime}-H_{1}+i_{\boldsymbol{\epsilon}}\right)^{-1} ;
\end{aligned}
$$

and $E$ is the total energy of the system.

The formal solution of $\mathrm{Eq} .(6)$ is:

$$
T=\left(1-V(r)^{-1}{ }^{r}\right.
$$

In the usual perturbation treatment, the factor $\left(1-V(x)^{-1}\right.$ is replaced by the first few terms of the geometric series.

$$
\left(1-V(r)^{-1}=\sum_{s=0}^{\infty}\left(V(i)^{s}\right.\right.
$$

For thermal neutron scattering from nuclei, this series converges very slowly at best, and perhaps it does not converge at all. Thus, for thermal neutrons, the Fermi approximation (1) has been used for Eq. (7).

However, this approximation has not been deduced in a systematic way for general scattering systems. The Fermi approximation has been deduced in at 
systematic way for scattering systems composed of one and two atoms, and the corrections have been calculated for these cases $(1,2)$.

We derive the Fermi approximation, for an arbitrary number of atoms in the scatterer, as the first term in a geometric series that is different from Eq. (8). To do this, let us consider the features of the neutron-nucleus potential, $V_{\alpha}\left(\mathbf{r}_{n}-\mathbf{r}_{\alpha}\right)$. The range of the potential $\left(\sim 10^{-13} \mathrm{~cm}\right)$ is very small compared to the average spacing between nuclei in normal matter $\left(\sim 10^{-8} \mathrm{~cm}\right)$. The depth of the potential is sufficiently great that equation (8) converges slowly or does not converge for thermal energies and $r_{n} \sim \mathbf{r}_{\alpha}$.

However, due to the short range of the potential, we can expand $T$ in a different geometric series that does converge rapidly. We write $V$ as the sum of two body potentials.

$$
T=\sum_{\alpha}(1-V G)^{-1} V_{\alpha}\left(\mathbf{r}_{n}-\mathbf{r}_{\alpha}\right) .
$$

Now, we add and subtract $V_{\alpha}\left(\mathbf{r}_{n}-\mathbf{r}_{\alpha}\right) G_{0}$ in $(1-V G)^{-1}$

$$
T=\sum_{\alpha}\left(1-V_{\alpha} G_{0}-\left\{V G-V_{\alpha} G_{0}\right\}\right)^{-1} V_{\alpha} ;
$$

where

$$
V_{\alpha}=V_{\alpha}\left(\mathbf{r}_{n}-\mathbf{r}_{\alpha}\right)
$$

and

$$
G_{0}=\left(-\left(\mathbf{p}_{n}{ }^{2} / 2 \mu_{\alpha}\right)+i \epsilon\right)^{-1}
$$

where $\mu_{\alpha}$ is the reduced mass of the neutron and the $\alpha^{\prime}$ th atom.

If $G \approx G_{0}$, then

$$
V G-V_{\alpha} G_{0} \approx \sum_{\beta}^{\prime} V_{\beta} G_{0}
$$

where $\sum_{\beta}{ }^{\prime}$ indicates the sum over all $\beta \neq \alpha$. Thus, we can expect that VG $-V_{\alpha} G_{0}$ will be small unless $\mathbf{r}_{n} \sim \mathbf{r}_{\beta}$, where $\beta \neq \alpha$. In this case, the $V_{\alpha}$ multiplying $(1-V G)^{-1}$ is zero since the range of $V_{\alpha}$ is much smaller than the separation of the atoms. From these arguments, we can expect that a series in the factor $\left\{V G-V_{\alpha} G_{0}\right\}$ will converge rapidly, and we can write Eq. (9) as a geometric series.

$$
T=\sum_{\alpha}\left(1-V_{\alpha} G_{0}\right)^{-1} \sum_{s=0}^{\infty}\left\{\left(V G-V_{\alpha} G_{0}\right)\left(1-V_{\alpha} G_{0}\right)^{-1}\right\}^{s} V_{\alpha} .
$$

If we retain only the first term in this series, we have:

$$
T^{0}=\sum_{\alpha} T_{\alpha}^{0}
$$


where

$$
T_{\alpha}{ }^{\prime}=\left(1-V_{\alpha}\left(r_{0}\right)^{-1} V_{\alpha} .\right.
$$

Notice that $T_{\alpha}{ }^{\prime \prime}$ is just the transition operator for a zero energy neutron scattering from a nucleus at $r_{\alpha}$. The matrix elements of this operator hetween neutron states are well known (.3).

$$
\left\langle\psi_{f}\left(\mathbf{r}_{n}\right)\left|T_{\alpha}^{0}\right| \psi_{i}\left(\mathbf{r}_{n}\right)\right\rangle=\left(2 \pi \hbar^{2} m\right) a_{\alpha} e^{i\left(\mathbf{k}_{1}-\mathbf{k}_{f}{ }^{\prime} \cdot \mathbf{r}_{i}\right.}
$$

where $a_{\alpha}$ is the bound atom scattering length for the $\alpha^{\prime}$ th atom. Taking matrix elements of $\mathrm{H}_{1}$. (12) between the states of the atoms, we have the fiermi approximation

$$
T_{j i}^{0}=\frac{2 \pi \hbar^{2}}{m} \sum_{\alpha}\left\langle\varphi_{j} \mid a_{\alpha} e^{i\left(\mathbf{k}_{i}-\mathbf{k}_{j}\right) \cdot \mathbf{r}_{\alpha r}} \varphi_{i}\right\rangle .
$$

If we retain the first two terms in the series, we have:

$$
T=\sum_{\alpha}\left(T_{\alpha}^{0}+T_{\alpha}{ }^{1}\right)
$$

where

$$
T_{\alpha}{ }^{1}=\left(1-V_{\alpha}\left(r_{i j}\right)^{-1}\left(I ^ { r } \left(r-V_{\alpha}^{r}\left(r_{0}\right)\left(1-V_{\alpha}^{r}\left(r_{H}\right)^{-1} T_{\alpha} .\right.\right.\right.\right.
$$

The matrix elements of $T_{\alpha}^{\prime}$ ' can be determined easily by including an intermediate set of the eigenstates of $\mathfrak{H}_{0}^{2}$.

$$
\begin{aligned}
& \left(T_{\alpha}{ }^{1}\right)_{j i}=\sum_{i, l}\left\langle\psi_{f} \varphi_{f}\right|\left(1-V_{\alpha}\left(T_{j i}\right)^{-1} V_{\alpha}\left|\psi_{j} \varphi_{l}\right\rangle \times\left\langle\psi_{j} \varphi_{j}\right|\left(1-V_{\alpha}\left(i_{i 1}\right)^{-1} \Gamma_{\alpha}^{*}\left|\psi_{i} \varphi_{i}\right\rangle\right.\right. \\
& \cdot\left(\left(_{j l}^{\prime}-G_{i n j}\right)+\sum_{j l} \sum_{\beta}^{\prime}\left\langle\psi_{j} \varphi_{f}\right|\left(1-I_{\alpha}\left(r_{i l)}\right)^{-1} V_{\beta}\left|\psi_{j} \varphi_{l}\right\rangle\right.\right. \\
& \times\left\langle\psi_{j \varphi l}\right|\left(1-I_{\alpha}\left(r_{i 1}\right)^{-1} I_{\alpha} \mid \psi, \varphi_{i}\right) r_{i, j}
\end{aligned}
$$

where

$$
G_{j l}=\left(E-\frac{\hbar^{2} \mathbf{k}_{j}^{2}}{2 m}-E_{l}+i \epsilon\right)^{-1}
$$

and

$$
G_{0 j}=\left(-\frac{\hbar^{\prime \prime} \mathbf{k}_{j}{ }^{2}}{2 \mu_{\alpha}}+i \epsilon\right)^{-1} .
$$

Applying Eq. (12), we have:

$$
\begin{aligned}
& \left(T_{\alpha}{ }^{1}\right)_{j i}=\sum_{j l}\left(\frac{2 \pi \hbar^{2}}{m} a_{\alpha}\right)^{2}\left\langle\varphi_{f}\left|e^{i\left(\mathbf{k}_{j}-\mathbf{k}_{j}\right) \cdot \mathbf{r}_{\alpha}}\right| \varphi_{l}\right\rangle\left\langle\varphi_{l} \mid e^{-i\left(\mathbf{k}_{j}-\mathbf{k}_{i}\right) \cdot r_{\sigma^{\prime}}}, \hat{\varphi}_{i}\right\rangle \\
& \times\left(G_{j l}-G_{i 0 j}^{\prime}\right)+\sum_{j l} \sum_{\beta}^{\prime} \frac{2 \pi \hbar^{2}}{m .} a_{\alpha}\left\langle\psi_{f} \varphi_{f}\right|\left(1-V_{\alpha}\left(G_{b i}\right)^{-1} V_{\beta}\left|\psi_{i} \varphi_{l}\right\rangle\right. \\
& X\left\langle\varphi_{i} \mid c^{-i\left(\mathbf{k}_{j}-\mathbf{k}_{1} \cdot \mathbf{r}_{12}\right.}: \varphi_{i}\right\rangle\left(\ell_{1}\right)
\end{aligned}
$$


The factor ( $\left.1-V_{\alpha} G_{0}\right)^{-1} V_{\beta}$ can be expanded similarly to Eq. (8) with only the first term retained since when $V_{\alpha}$ is large $V_{\beta}$ is zero. Including this approximation and inserting Eq. (16) into the expression for the matrix elements of Eq. (14), we obtain

$$
\begin{aligned}
& T_{f i}=\frac{2 \pi \hbar^{2}}{m} \sum_{\alpha}\left\langle\varphi_{j}\left|a_{\alpha} e^{i\left(\mathbf{k}_{i}-\mathbf{k}_{f}\right) \cdot \mathbf{r}_{\alpha}}\right| \varphi_{i}\right\rangle \\
& +\sum_{\alpha} \sum_{j l}\left(\frac{2 \pi \hbar^{2}}{m} a_{\alpha}\right)^{2}\left\langle\varphi_{f}\left|e^{i\left(\mathbf{k}_{j}-\mathbf{k}_{f}\right) \cdot \mathbf{r}_{\alpha}}\right| \varphi_{l}\right\rangle\left\langle\varphi_{l}\left|e^{-i\left(\mathbf{k}_{j}-\mathbf{k}_{i}\right) \cdot \mathrm{r}_{\alpha^{\prime}}}\right| \varphi_{i}\right\rangle\left(G_{j l}-G_{0 j}\right) \\
& \quad+\sum_{\alpha} \sum_{j l} \sum_{\beta}^{\prime} \frac{2 \pi \hbar^{2}}{m} a_{\alpha} b_{\beta}\left\langle\varphi_{f}\left|e^{i\left(\mathbb{k}_{j}-\mathbf{k}_{f}\right) \cdot \mathbf{r}_{\beta}}\right| \varphi_{l}\right\rangle\left\langle\varphi_{l}\left|e^{-i\left(\mathbf{k}_{j}-\mathbf{k}_{i}\right) \cdot \mathbf{r}_{\alpha^{\prime}}}\right| \varphi_{i}\right\rangle G_{j l}
\end{aligned}
$$

where

$$
b_{\beta}=\int e^{i\left(\mathrm{k}_{j}-\mathrm{k}_{j}\right) \cdot \mathbf{r}} V_{\beta}(\mathbf{r}) d^{3} r^{3}
$$

or, since $V_{\beta}$ is a short range potential,

$$
b_{\beta}=\int V_{\beta}(\mathrm{r}) d^{3} r
$$

\section{A SINGLE BOUND PROTON}

Now, we will specialize these results to the case of neutron scattering from a single proton bound in an otherwise inert molecule. In this case the interaction $V\left(\mathbf{r}_{n}-\mathbf{r}_{p}\right)$ will contain a single two-body term. We will perform our analysis in the center of mass system of the neutron and the molecule, and we will assume the energy of the center of mass is zero.

$$
G_{0}=\left(-\mathrm{p}_{n}{ }^{2} / m+i \epsilon\right)^{-1}
$$

If we take the masses of the neutron and proton to be equal, the reduced mass is just one-half the neutron mass.

In this case the matrix elements of $\left(1-V G_{0}\right)^{-1} V$ between free neutron states are

$$
\left\langle e^{i \mathbf{k}_{f} \cdot \mathbf{r}_{n}}\left|\left(1-V G_{0}\right)^{-1} V\right| e^{i \mathbf{k}_{i} \cdot \mathbf{r}_{n}}\right\rangle=\frac{4 \pi \hbar^{2}}{m} a^{\prime} e^{i\left(\mathbf{k}_{i}-\mathbf{k}_{f}\right) \cdot \mathbf{r}_{p}}
$$

where $a^{\prime}$ is the neutron-proton free atom scattering length. Following an analogous procedure to that of section II, we obtain the following expression for the $T$-matrix, including the Fermi approximation and the first order correction.

$$
\begin{aligned}
T_{j i}=\frac{4 \pi \hbar^{2}}{m} a^{\prime} & \left\{\left\langle\varphi_{f}\left|e^{i\left(\mathbf{k}_{i}-\mathbf{k}_{j}\right) \cdot \mathbf{r}_{p}}\right| \varphi_{i}\right\rangle\right. \\
& \left.+\frac{4 \pi \hbar^{2}}{m} a^{\prime} \sum_{j l}\left\langle\varphi_{j}\left|e^{i\left(\mathbf{k}_{j}-\mathbf{k}_{j}\right) \cdot \mathbf{r}_{p}}\right| \varphi_{l}\right\rangle\left\langle\varphi_{l}\left|e^{-i\left(\mathbf{k}_{j}-\mathbf{k}_{i}\right) \cdot \mathbf{r}_{p}}\right| \varphi_{i}\right\rangle\left(G_{j l}-G_{0 j}\right)\right\}
\end{aligned}
$$


where

$$
\begin{aligned}
& G_{j l}=\left(E-E_{l}-\frac{\hbar^{2} \mathbf{k}_{j}^{2}}{2 \mu}+i \epsilon\right)^{-1} \\
& G_{0 j}=\left(-\frac{\hbar^{2} \mathbf{k}_{j}^{2}}{m}+i \epsilon\right)^{-1}
\end{aligned}
$$

and where $\mu$ is the reduced mass of the molecule and the neutron; and $\varphi_{i}\left(r_{p}\right)$ is the energy eigenfunction of the molecule.

The sum over the free neutron states is in reality an integral over the three dimensional momentum continuum.

$$
\sum_{j} \rightarrow \int \frac{d^{3} k_{j}}{(2 \pi)^{3}}
$$

making this substitution, and utilizing the following well known fourier integral,

$$
\int d^{3} k e^{i \mathbf{k} \cdot \mathbf{R}} \frac{1}{a^{2}-k^{2}+i \epsilon}=-\frac{2 \pi^{2}}{R} a^{i a h r}
$$

wo obtain the following result for the $T$-matrix.

$$
\begin{aligned}
& T_{i i}=\frac{4 \pi \hbar^{2}}{m !} a^{\prime}\left\{\left\langle\varphi_{j}\left|c^{\left.i l \mathbf{k}_{i}-\mathbf{k}_{i}\right) \cdot \mathbf{r}_{2}}\right| \varphi_{i}\right\rangle\right. \\
& -a^{\prime} \sum_{l} \iint d^{3} r_{p} d^{3} r_{p}{ }^{\prime} \varphi_{f}^{*}\left(\mathbf{r}_{p}\right)_{\varphi l}\left(\mathbf{r}_{p}\right) e^{-i \mathbf{k}_{j} \cdot \mathbf{r}^{\prime \prime} \varphi_{l}{ }^{*}\left(\mathbf{r}_{p}{ }^{\prime} \mid \varphi_{i}\left(\mathbf{r}_{p}{ }^{\prime}\right)\right.}
\end{aligned}
$$

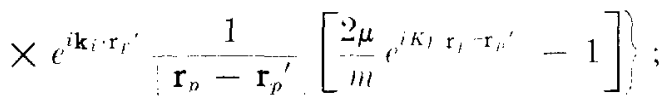

where

$$
K_{l}=\left\{\frac{2 \mu}{\hbar^{2}}\left(E-E_{l}\right)\right\}^{\mid 2}
$$

This is exactly the result Lippmann and Schwinger obtained with a somewhat longer derivation (3).

The second term in Eq. (20) has been shown to be at most a fraction of a per cent of the first term (2). If there is any case where the correction to the Fermi approximation is significant, we would not expect that it is this one. In fact, Davydov and Mel'nichenko ( 4 ) have shown that the correction vanishes for zero energy scattering from a free proton at rest. This is not surprising since the lermi approximation was constructed to give the correct result in this cance.

\section{THE MAGNITU])E OF THE FIRST ORINR CORRECTION}

Although the lermi approximation has been shown to be valid for a single scattering atom, it does not predict any multiple scattering effects. Thus, it 
cannot adequately account for a system in which multiple scattering is important. In particular, it is of interest to consider the possibility of multiparticle excitations (i.e., a transfer of energy between two or more atoms and a scattered neutron). In the Fermi approximation, such processes depend on the dynamical correlation of the atoms. However, if we retain the higher order terms in Eq. (17), such processes are possible even in systems with no dynamical correlation. It is entirely possible that in a liquid or dense gas where the dynamical correlation is small the higher order terms could give the dominant contribution to multiparticle excitations. Some of these considerations have been presented previously by Nelkin (5).

Thus, we will evaluate Eq. (17) approximately to obtain an idea of the importance of multiple scattering. Ferziger and Leonard $(6)$ have made a study of multiple scattering in the static approximation, but there has been no previous work done for a dynamical scattering system. Consider a diagonal element of the T-matrix in the limit that $k_{i}=k_{f}=E=E_{l}=0$. The second term in Eq. (17) vanishes and the sum over $l$ in the third term can be carried out, giving unity. In this approximation, $T_{i i}$ is

$$
T_{i i}=\frac{2 \pi \hbar^{2}}{m} \sum_{\alpha} a_{\alpha}\left\{1+\sum_{\beta}^{\prime} \sum_{j} b_{\beta} G_{0 j}\left\langle\varphi_{i}\left|e^{i \mathbf{k}_{j} \cdot\left(\mathbf{r}_{\beta}-\mathrm{r}_{\alpha}\right)}\right| \varphi_{i}\right\rangle\right\}
$$

We can evaluate the $\sum_{j}$ as an integral over the momentum $\mathbf{k}_{j}$ in exactly the same way as was done in Section III, giving

$$
T_{i i}=\frac{2 \pi \hbar^{2}}{m} \sum_{\alpha} a_{\alpha}\left\{1+\sum_{\beta}^{\prime} \frac{b_{\beta} m}{2 \pi \hbar^{2}}\left\langle\varphi_{i}\left|\frac{1}{\left|\mathbf{r}_{\beta}-\mathbf{r}_{\alpha}\right|}\right| \varphi_{i}\right\rangle\right\}
$$

The matrix element in this expression is the average of the inverse distance between the $\alpha$ th and $\beta$ th atoms. Let us approximate this quantity by the inverse of the average distance, $d_{\alpha \beta}$.

$$
\left\langle\varphi_{i}\left|\left(1 /\left|\mathbf{r}_{\beta}-\mathrm{r}_{\alpha}\right|\right)\right| \varphi_{i}\right\rangle \approx 1 / d_{\alpha \beta} .
$$

The magnitude of $b_{\beta} m / 2 \pi \hbar^{2}$ is on the order of $10^{-12} \mathrm{~cm}$, and the distance between neighboring atoms is $d \sim 10^{-8} \mathrm{~cm}$. Thus, the largest correction terms in Fq. (21) are about $10^{-4}$ of the Fermi approximation. However, the number of terms of a given size increase as the square of $d_{\alpha \beta}$, and by making a scatterer sufficiently large one can always make the correction significant. In fact, this approximate correction increases with the number of scattering atoms sufficiently rapidly that it dominates the lermi approximation for scatterers of macroscopic size. This rather striking result is due to the fact that we evaluated the correction in the limit of zero (not thermal) energy. In the zero energy limit, the neutron wavelength is infinite, and one can consider that the neutron interacts with the scattering system as a whole rather than the individual atoms 
separately. The Fermi approximation treats the scattering in terms of the individual neutron-nucleus interactions, and we would thus not expect it to apply for zero energy and large scattering systems.

This effect would only be observed for neutron energies considerably below thermal and then only for scatterers of nearly macroscopic size.

\section{DISCUSAION}

If we use $\mathrm{F}$. (19) to perform the sum over $j$ in $\mathrm{F}_{(1 .} 117$ ), we obtain $T_{f}$, in a form that is similar to the Lippmanm-Schwinger result $[1 \%(20)]$.

$$
\begin{aligned}
& T_{j i}=\frac{2 \pi \hbar^{2}}{m} \sum_{\alpha}\left\langle\varsigma_{j}\left|a_{\alpha} e^{i\left(\mathbf{k}_{i}-\mathbf{k}_{j}\right) \cdot \mathbf{r}_{\alpha}}\right| \varphi_{i}\right\rangle-\sum_{\alpha} \sum_{i} \frac{2 \pi h^{2}}{m !} a_{\alpha}^{2} \\
& \times \int d^{\prime \prime}{ }_{1} \cdots d^{3} \gamma_{N} d^{3} v_{1}^{\prime} \cdots d^{3} \gamma_{N}^{\prime} \varphi_{j}^{*}\left(\mathbf{r}_{1}, \cdots, \mathbf{r}_{N}\right) \varphi_{l}\left(\mathbf{r}_{1}, \cdots, \mathbf{r}_{N}\right) e^{-i \mathbf{k}_{/} \cdot \mathbf{r}_{1}}
\end{aligned}
$$

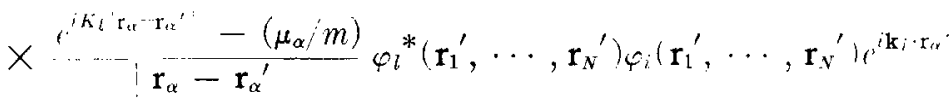

$$
\begin{aligned}
& -\sum_{\alpha} \sum_{i} \sum_{\beta}^{\prime} a_{\alpha} b_{\beta} \int d^{3} r_{1} \cdots d^{3} r_{N} d^{3}{ }^{\prime}{ }_{1}^{\prime} \cdots d^{*} r_{N}{ }^{\prime} \rho_{l}^{*}\left(\mathbf{r}_{1}, \cdots, \mathbf{r}_{N}\right) \\
& \times \varphi_{l}\left(\mathbf{r}_{1}, \cdots, \mathbf{r}_{N}\right) e^{-i \mathbf{k}_{j} \cdot \mathbf{r}_{\beta}} \frac{e^{i K_{l}\left|\mathbf{r}_{\beta}-\mathbf{r}_{\alpha}{ }^{\prime}\right|}}{\left|\mathbf{r}_{\beta}-\mathbf{r}_{\alpha}{ }^{\prime}\right|} \varphi_{l}{ }^{*}\left(\mathbf{r}_{1}{ }^{\prime}, \cdots, \mathbf{r}_{N}{ }^{\prime}\right)_{\varphi_{i}}\left(\mathbf{r}_{1}{ }^{\prime}, \cdots, \mathbf{r}_{N}{ }^{\prime} k^{i \mathbf{k}_{1} \cdot \mathbf{r}_{1 \ell}{ }^{\prime}}\right.
\end{aligned}
$$

where

$$
K_{l}=\left\{\frac{2 m}{\hbar^{2}}\left(E-E_{l}\right)\right\}^{1: 2}
$$

The important difference between the Lippmann-Schwinger lesult and $\mathrm{F}(1.22)$ is that $\mathrm{Fq}$. ( 22 ) has been derived for a scattering system with an arbitrary number of atoms. The Lippmann-Schwinger result is derived for a single scattering atom, although Lippmann generalizes the result to include two scattering atoms.

We have made a rather crude estimation of the magnitude of the correction term in which it was shown to be of order $10^{-4}$ of the lermi approximation. However this estimate depends critically on the size of the scatterer, and the neutron energy. The correction will be calculated in more detail in a future work. Also, as we pointed out in Section IV, the multiparticle excitations can provide information about the dynamical correlation of the atoms. Before undertaking an analysis to determine the dynamical correlation, it certainly will be necessary to compute the correction given by $\mathrm{Eq} .(22)$.

The subtraction device used in writing Eq. (10) may have more general application than thermal neutron scattering. For example, the Meson theory of muclear forces predicts the low energy mucleon-mucleon interaction quite ar. 
curately from the low order perturbation theory terms. However, attempts to calculate the higher order corrections have been unsuccessful, and there is a convergence problcm due to the large coupling constant. It may be that the application of a procedure similar to the one used here would help clarify this situation.

\section{ACKNOWLedgments}

The author wishes to thank Professor P. F. Zweifel and Professor R. K. Osborn for many stimulating discussions.

RECEIVED: May 13, 1963

\section{REFERENCES}

1. E. Fermi, Ricerca Sci. 7, 13 (1936).

2. G. Breit et al. Phys. Rev. 71, 215 (1917); ibid. 71, 232 (1947); ibid. 72, 516 (1947); B. A. Lippmann and J. Schwinger, Phys. Rev. 79, 469, 481 (1950); J. P. Plemmer and G. C. Summerfield, Phys. Rev. 131, 1153 (1963).

3. B. A. Lippmann and J. Schwinger, Phys. Rev. 79, 469, 481 (1950).

4. A. S. Davydov and D. M. Mel'nichenko, Zh. Eksperim. Teor. Fiz. 32, 941 (1957).

5. M. Nelkin, private communication.

6. J. Ferziger and A. Leonard, Phys. Rev. 128, 2188 (1962). 